\title{
Natural convection induced by an optically fabricated and actuated microtool with a thermoplasmonic disk
}

Engay, Einstom; Bunea, Ada-loana; Chouliara, Manto; Bañas, Andrew Rafael; Glückstad, Jesper

Published in:

Optics Letters

Link to article, DOI:

10.1364/OL.43.003870

Publication date:

2018

Document Version

Version created as part of publication process; publisher's layout; not normally made publicly available

Link back to DTU Orbit

Citation (APA):

Engay, E., Bunea, A-I., Chouliara, M., Bañas, A. R., \& Glückstad, J. (2018). Natural convection induced by an optically fabricated and actuated microtool with a thermoplasmonic disk. Optics Letters, 43(17), 3870-3873. https://doi.org/10.1364/OL.43.003870

\section{General rights}

Copyright and moral rights for the publications made accessible in the public portal are retained by the authors and/or other copyright owners and it is a condition of accessing publications that users recognise and abide by the legal requirements associated with these rights.

- Users may download and print one copy of any publication from the public portal for the purpose of private study or research.

- You may not further distribute the material or use it for any profit-making activity or commercial gain

- You may freely distribute the URL identifying the publication in the public portal 


\title{
Natural convection induced by an optically fabricated and actuated microtool with a thermoplasmonic disk
}

\author{
Einstom Engay, ${ }^{1}$ (i) Ada-loana Bunea, ${ }^{1, *}$ (i) Manto Chouliara, ${ }^{1}$ Andrew Bañas, ${ }^{1}$ and \\ Jesper GlüCKSTAD ${ }^{1,2}$ \\ ${ }^{1}$ DTU Fotonik, Department of Photonics Engineering, Technical University of Denmark, Orsted Plads 343, DK-2800 Kongens Lyngby, Denmark \\ ${ }^{2}$ e-mail: jesper.gluckstad@fotonik.dtu.dk \\ *Corresponding author: adabu@fotonik.dtu.dk
}

Received 15 June 2018; revised 18 July 2018; accepted 18 July 2018; posted 19 July 2018 (Doc. ID 335398); published 0 MONTH 0000

Two-photon polymerization was employed for fabricating microtools amenable to optical trapping and manipulation. A disk feature was included as part of the microtools and further functionalized by electron-beam deposition. The nanostructured gold layer on the disk facilitates off-resonant plasmonic heating upon illumination with a laser beam. As a consequence, natural convection characterized by the typical toroidal shape resembling that of RayleighBénard flow can be observed. A velocity of several $\mu \mathrm{m} \cdot \mathrm{s}^{-1}$ is measured for $2 \mu \mathrm{m}$ microspheres dispersed in the surroundings of the microtool. To the best of our knowledge, this is the first time that thermoplasmonic-induced natural convection is experimentally demonstrated using a mobile heat source. (๑) 2018 Optical Society of America

OCIS codes: (120.6810) Thermal effects; (310.1860) Deposition and fabrication; (140.7010) Laser trapping; (230.4000) Microstructure fabrication.

https://doi.org/10.1364/OL.99.099999

Recent developments in photolithographic techniques have widened fabrication possibilities and, thus, the applications of microstructures that are now becoming ubiquitous in scientific explorations in the micro- and nano-regimes. Microtools can increase the efficiency of processes that are otherwise difficult to achieve with conventional "macrotools" due to the inherent disparity in size between the macrotools and certain samples and specimens. Furthermore, microstructures can act as a mediator between a specimen and an actuator to minimize damage, especially when dealing with biological samples [1].

Two-photon polymerization (2PP) is a microfabrication technique that relies on the non-linear interaction between a tightly focused femtosecond pulsed light and a photosensitive resin. Two-photon absorption is initiated within the beam's focal volume and leads to the polymerization of the photoresist in the target region, while minimizing the polymerization in the out-of-focus regions [2]. A near-infrared (NIR) beam is usually employed to trigger crosslinking, since most resins are transparent in the NIR region, allowing the beam to focus with minimal intensity loss through the photoresist volume. The focused beam is then $3 \mathrm{D}$ scanned in the photoresist, which leads to polymerization along the beam's path and, thus, enables the fabrication of microstructures. Direct laser writing (DLW) using 2PP has been employed for the fabrication of microfluidic devices [3], implantable biomedical devices [4], and hydrogel scaffolds for tissue engineering [5]. DLW is extremely advantageous for rapid prototyping, as it allows fast design and fabrication iterations.

Along with topological design optimization, the functionalization of microtools by chemical or physical treatment allows them to be tailored to more specific tasks. Remote manipulation of microtools can be done by optical trapping, while additional light-matter interaction mechanisms can be used to initiate the intended function of the microtools. In most cases, the trapping beams are spatially separated from the illumination beam used to trigger the additional effect, making them independently configurable. Successful demonstrations of manipulating functionalized microtools include indirect optical manipulation of live cells $[6,7]$, puncturing of cells with embedded carbon nanotubes [8], light confinement and guiding to inaccessible areas in microfluidics [9], rotary motion of micro-rotors by transfer of linear momentum [10], spin angular momentum [11], and orbital angular momentum [12]. Targeted material delivery has been demonstrated by embedding a metal layer inside the body of a hollow microtool, which enabled a draw-and-eject function via thermally induced fluid motion [13].

Under illumination, metallic nanostructures enhance lightto-thermal energy conversion via plasmonic absorption, which makes them viable heat sources in the nano- and micro-regimes. The field of thermoplasmonics has been growing continuously since the early $2000 \mathrm{~s}$ when plasmonic heating was demonstrated for photothermal microscopy [14] and photothermal therapy [15]. Recently, thermoplasmonic structures have been employed for different applications such as localized optical heating [16], trapping [17,18], and biosensing [19]. 
In this Letter, we report the fabrication of a microtool with a thermoplasmonic disk. Using spherical trapping handles, the microtool can be moved to a target location, after which the heating can be activated on demand by a user. The increase in local temperature manifests in thermophoretic effects and the occurrence of natural convection. We are especially interested in an enhanced convection, as this can lead to increased mass flow rates, which are at the same time directed. This is of great interest in lab-on-a-chip systems, as it provides a fundamental understanding of energy conversion on the microscale, and potentially enables direct applications for material delivery and micro-mixing, which are both challenging in microfluidic systems. The generation of plasmon-induced convection is experimentally demonstrated. To the best of our knowledge, this is the first time the actual convection flow induced by thermoplasmonics with the aid of a mobile heat source is reported.

The microtools are fabricated by DWL using a 2PP-based microfabrication system (Nanoscribe Photonic Professional GT, Nanoscribe GmbH, Germany). The 3D design is done in SolidWorks and then transferred to the DeScribe software, where it is digitally sliced in 2D layers and, subsequently, transformed into hatched lines. The fabrication involves scanning the beam focus layer by layer along the hatched lines by moving the stage where the substrate is mounted. We set the slicing and hatching distances to $200 \mathrm{~nm}$ and fixed the power scaling to correspond to a $16 \mathrm{~mW}$ average power at the aperture objective. The stage velocity was set to $200 \mu \mathrm{m} \cdot \mathrm{s}^{-1}$. We used the Nanoscribe IPL-80 as photoresist to print on glass substrates. Our microtool has three spherical trapping handles $(8 \mu \mathrm{m}$ diameter) and a disk (10 $\mu \mathrm{m}$ diameter, $2 \mu \mathrm{m}$ thickness). The microtool was designed in this way to attain minimal size while allowing for stable optical trapping through its spherical handles. To allow selective metal coating of the disk, a mask covering the spherical handles and connecting parts was designed and 3D-printed on top of the microtools. The printing of nine microtools with masks takes approximately $4 \mathrm{~h}$. After fabrication, the glass substrate containing the printed microtools is immersed for $20 \mathrm{~min}$ in isopropanol to ensure the removal of uncrosslinked photoresist. For fabricating metal-coated microtools, $1 \mathrm{~nm}$ of titanium as an adhesive layer followed by a $10 \mathrm{~nm}$ layer of gold were deposited on the disks by a physical vapor electron-beam (e-beam) deposition (Wordentec QCL800). The metal layer obtained after gold e-beam deposition is exploited for off-resonant plasmonic heating. After metal coating, the microtools were harvested from the substrate using a syringe with a $100 \times 100 \mu \mathrm{m}^{2}$ square capillary tube, as described in Ref. [13]. The microtools were then transferred into a Hellma cuvette $\left(250 \times 250 \mu \mathrm{m}^{2}\right.$ inner cross section, $20 \mathrm{~mm}$ length) containing $0.125 \%$ polystyrene microspheres ( $2 \mu \mathrm{m}$ diameter Polybead ${ }^{\circledR}$ Microspheres, PolySciences, Inc.) and $1 \%$ Tween 80 in Milli-Q water $(18.2 \mathrm{M} \Omega \cdot \mathrm{cm})$.

Optical trapping was performed in our Biophotonics Workstation (BWS) that employs counter-propagating (CP) beams to control and actuate particles [Fig. 1(a)] [20,21]. A CP beam relies on scattering forces for trapping instead of the gradient force used in more common optical tweezers [22]. As such, the $\mathrm{CP}$ trapping geometry does not require high numerical aperture (NA) objectives and, thus, accommodates a long working distance, which allows for the insertion of an additional objective for side imaging. In our BWS, the CP beams are generated using a proprietary illumination module and a

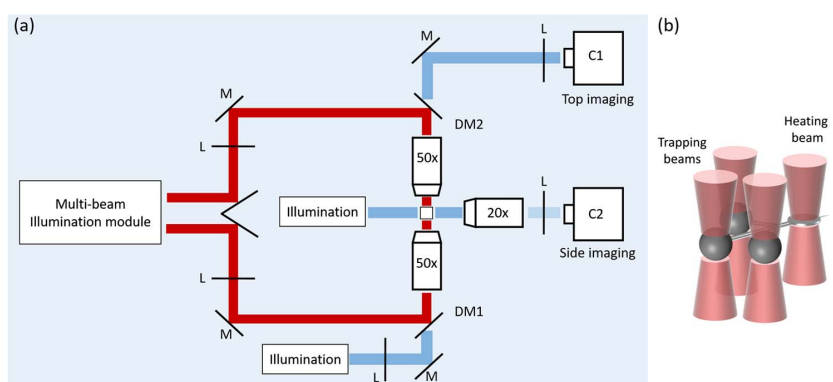

Fig. 1. (a) Schematic diagram of the Biophotonics Workstation. A $1070 \mathrm{~nm}$ laser is used for both optical trapping and heating. (b) Schematic representation of a microtool with CP trapping and heating beams.

continuous-wave NIR laser (IPG Photonics, $\lambda=1070 \mathrm{~nm}$, $40 \mathrm{~W}$ maximum input power). The beams are then relayed onto the sample using two opposing objectives (Olympus LMPLN, 50× IR, WD $=6.0 \mathrm{~mm} \mathrm{NA}=0.55)$. A LabViewbased graphical user interface (GUI) is used to generate multiple traps displayed as overlay graphics on real-time images acquired with the top camera. Displacement of the microtool in the $x-y$ plane is executed by dragging the trap overlays to the desired location, while manipulation on the $z$-axis is performed by changing the intensity ratio of the top and bottom beams. The size of the beam spots can also be adjusted from the GUI. In addition to the top-view recording, side imaging is available in the setup using a $20 \times$ microscope objective (Mitutoyo Plan Apo, 20×, WD $=20 \mathrm{~mm}$, NA = 0.42). In our experiments, three $\mathrm{CP}$ beams were generated for trapping the microtool, and a fourth CP beam was directed at the disk to induce heating [Fig. 1(b)]. The trapping and heating beam spot sizes were set to $10 \mu \mathrm{m}$. In our experiments, the total optical power reaching the microtool is in the order of tens of milliwatts equally divided into the heating and trapping CP beams. The uncertainty arises from the losses in the measurement system. One of the challenges in illuminating the coated disk is the recoil of the microtool due to beam reflection, but this is minimized by trapping the microtool with the aid of the spherical handles.

The videos used for particle tracking consist of 840 frames recorded at 21 frames per second, corresponding to a duration of $40 \mathrm{~s}$. The videos were recorded using the side-view camera from the time the heating beam was turned on. Particle tracking was performed using the Spot Tracking plugin in the Icy image analysis software. Only the particles that remain in focus in the imaging plane for at least 525 frames were taken into account for subsequent data analysis in order to minimize calculation errors due to out-of-plane displacements. The first 525 frames $(25 \mathrm{~s})$ of all tracks obtained were used for further data processing. The mean square displacements (MSDs) of individual particles were calculated from the coordinates of the particles using the freely available Matlab tool msdanalyzer [23]. In our experiments, we analyze and compare particle trajectories for microtools with both uncoated and coated disks.

Examples of the 3D-printed microtools employed in this Letter are shown in Fig. 2. Each microtool has three spherical handles that allow optical trapping and manipulation. As seen in Fig. 2(b), the mask printed on top of the microtools during
$\mathrm{F} 1: 1$

$\mathrm{F} 1: 2$

F1:3

$\mathrm{F} 1: 4$ 


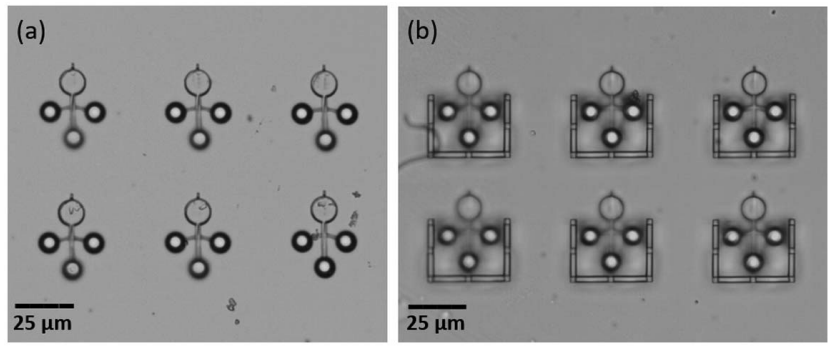

Fig. 2. $40 \times$ bright field images of 3D-printed microtools (a) without and (b) with masks for subsequent metal coating.

the fabrication process selectively exposes the disk feature for metal coating.

Control experiments were performed with uncoated microtools. Figure 3 shows one uncoated microtool just before (a), and $2 \mathrm{~s}$ (b) and $240 \mathrm{~s}$ (c) after the heating beam was turned on. The microspheres located in the immediate vicinity of the trapping spheres and disk were trapped when the laser was turned on. Leaving the trapping and heating beams on for four minutes resulted in some additional aggregation of microspheres around the trapping spheres and disk, but no significant motion was observed for microspheres farther from the microtool. Although the uncoated disk can serve as a heat source by light absorption, the generated heat is not enough to effect global motion in the fluid.

In contrast, illumination of the gold-coated microtools resulted in the immediate expulsion of microspheres away from the disk, as can be seen from Figs. 3(d)-3(f). This can be explained by the increase in temperature around the disk upon illumination. The metal-coated disk serves as an efficient heat source by virtue of off-resonant plasmonic heating. Energy absorption is greatly enhanced with the aid of the plasmonic nanostructures, which have been previously used as heat sources [24]. Due to thermophoretic effects, the microspheres that were initially near the disk tend to move in the direction of the temperature gradient-from the hotter disk to the cooler

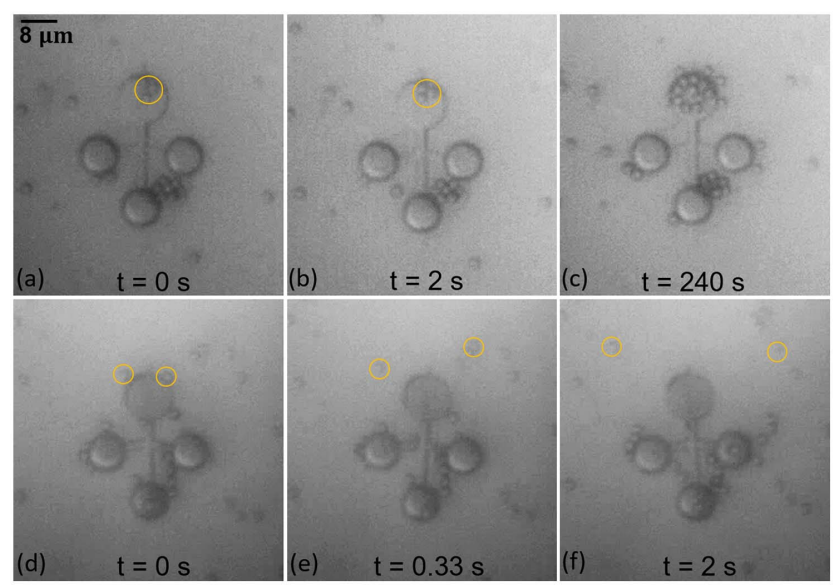

after turning on the heating beam. The microspheres slowly aggregate around the disk in the presence of the uncoated microtool (a)-(c), but are rapidly pushed away in the presence of the gold-coated microtool (d)-(f). area around it. Factors affecting thermophoresis have been exthe uncoated (a) and coated (b) microtools when the disk is

$$
\rho(t)=4 D t+v^{2} t^{2},
$$
tensively studied [25], as well as its applications to thermophoretic trapping of nano-objects by plasmonic heating $[18,26]$.

For the gold-coated microtools, thermophoretic diffusion is a consequence of disk heating. When the disk is illuminated, it heats up by plasmonic absorption and generates a convection flow that drags particles towards the microtools which leads to their movement from the bottom to the top of the microchamber. Thermophoresis prevents the microspheres from getting into the immediate surroundings of the disk.

Figure 4 shows a schematic of the microsphere motion for actuated using the heating beam.

For characterizing particle motion, the MSDs were calculated from the side-view recordings. A linear MSD versus time relation indicates that the particles are in Brownian motion [27-29], in which case the MSD at any given time is given by Einstein's equation for Brownian motion in 2D:

$$
\rho(t)=4 D t
$$

where $\rho(t)$ is the MSD, $D$ is the diffusion coefficient, and $t$ is the time. In the case of directed motion, the MSD increases faster with time, which manifests in a positive curvature in the MSD versus time plot. Thus, in the case of a directed trajectory, the particle MSD is given by [29]

where $v$ is the drift velocity, and the other parameters are the same as in Eq. (1).

Individual particle trajectories over a period of $40 \mathrm{~s}$ in the presence of an uncoated tool are shown in Fig. 5(a). From the linear fit of the average particle MSD versus time experimental data points, the diffusion coefficient was calculated to be $2.98 \pm 1.73 \mu \mathrm{m}^{2} \mathrm{~s}^{-1}$. The highly erratic individual particle trajectories and the linear increase of the average MSD with time [Fig. 5(c)] indicate that, in this case, the motion of the microspheres is highly associated to Brownian motion. Sedimentation due to gravity and convection induced by heating the disk do not have a significant effect on particle motion in the bulk of the solution. While particles near the beams will tend to slowly move towards the disk due to the gradient force introduced by the heating beam, this does not lead to any collective movement of particles towards the microtool within the time period considered.

For the coated microtool, illumination of the disk led to natural convection characterized by the typical toroidal shape resembling that of Rayleigh-Bénard flow, as depicted in the particle trajectories shown in Fig. 5(b) and in the flow pattern shown in Fig. 5(d). The local increase in temperature leads to a

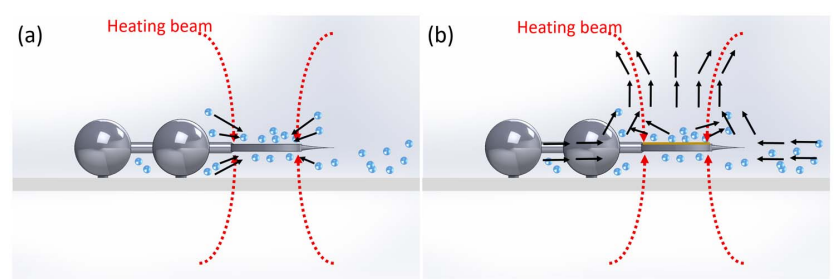

Fig. 4. Schematic of microsphere movement after the heating beam is turned on to illuminate the disk of (a) uncoated and (b) gold-coated microtools.
F4:1

$\mathrm{F} 4: 2$

$\mathrm{F} 4: 3$ 
(a)
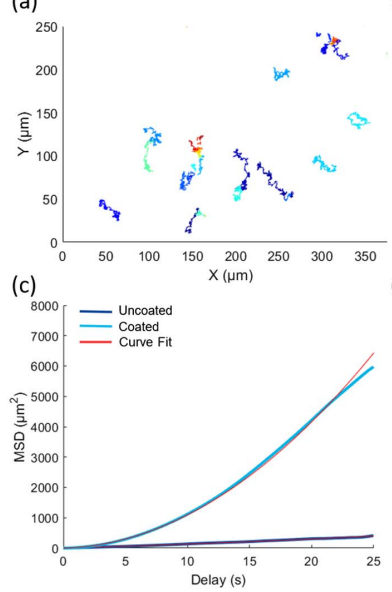

(b)

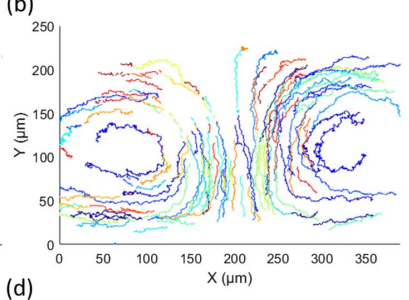

(d)

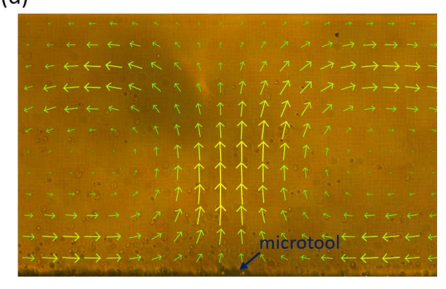

F5:1

$\mathrm{F} 5: 2$

F5:3

Fig. 5. Individual particle trajectories calculated from the side-view imaging in the presence of (a) uncoated and (b) gold-coated microtools for a duration of $40 \mathrm{~s}$. (c) MSD comparison for coated and uncoated microtools. (d) Flow pattern in the measurement chamber generated by heating a gold-coated disk microtool.

decrease in the fluid density around the disk, which then generates natural convection currents. Distant microspheres outside the field of view were dragged by the fluid flow towards and away from the microtool radially and vertically. This " $m i-$ gration" cannot be associated with the gradient force of the optical traps alone, as demonstrated by the reference experiments using uncoated microtools. By fitting the MSD versus time curve according to Eq. (2), we calculated a diffusion coefficient of $2.74 \pm 0.11 \mu \mathrm{m}^{2} \mathrm{~s}^{-1}$, which is in good agreement with that of microspheres in the presence of the uncoated microtool. From the same fit, the average particle velocity was found to be $3.14 \pm 0.02 \mu \mathrm{m} \mathrm{s}^{-1}$. This average particle velocity is higher or comparable with previously reported results $[26,30,31]$. The convection pattern we obtained [Fig. 5(d)] is similar to the simulation results presented in Roxworthy et al. for an array of gold bowtie nanoantennas (BNAs) patterned on top of an indium-tin-oxide (ITO) layer [31]. However, in their case, the temperature increase was associated with the optical absorption of the ITO layer in NIR, while the BNAs merely had the role of enhancing the heat absorption of ITO. In our case, the temperature increase arises from the Joule effect on the gold nanolayers.

Microtools with gold-coated disks were successfully fabricated and employed for localized heating in a straight and closed microchannel. Spherical handles included as part of the microtool design enable optical trapping and manipulation of the microtools, which further facilitates precise actuation. By illuminating the disk with a laser beam, we demonstrate off-resonant plasmonic heating, which leads to natural convection in the system. Upon short-term illumination of the gold-coated disk $(<2 s)$, nearby microspheres are immediately repelled due to the creation of thermophoretic gradients. Further heating of the disk induces a natural convective flow that spans several hundreds of micrometers. Illumination of similar microtools without gold coating on the disk does not lead to any significant motion in the system. This suggests that the natural convective flow observed can be attributed to the off-resonant plasmonic heating of the disk, rather than any other effects related to the presence of the microtool or laser beam. To the best of our knowledge, this represents the first direct observation of natural convection induced by means of off-resonant plasmonic heating with the aid of a mobile heat source. This approach could prove valuable for understanding and controlling micro-mixing and material delivery in microfluidic devices.

297

298

299

300

301

302

Funding. Novo Nordisk Fonden (NNF) 4

(NNF16OC0021948).

303 304

\section{REFERENCES}

1. J. Glückstad and D. Palima, Light Robotics: Structure-Mediated Nanobiophotonics (Elsevier, 2017).

2. S. Maruo, O. Nakamura, and S. Kawata, Opt. Lett. 22, 132 (1997).

3. L. Amato, Y. Gu, N. Bellini, S. M. Eaton, G. Cerullo, and R. Osellame, Lab Chip 12, 1135 (2012).

4. A. I. Son, J. D. Opfermann, C. McCue, J. Ziobro, J. H. Abrahams, K. Jones, P. D. Morton, S. Ishii, C. Oluigbo, A. Krieger, J. S. Liu, K. Hashimoto-Torii, and M. Torii, Sci. Rep. 7, 1 (2017).

5. S. You, J. Li, W. Zhu, C. Yu, D. Mei, and S. Chen, J. Mater. Chem. B 6 , 2187 (2018)

6. G. Vizsnyiczai, B. L. Aekbote, A. Buzás, I. Grexa, P. Ormos, and L. Kelemen, Proc. SPIE 9922, 992216 (2016).

7. E. Avci, M. Grammatikopoulou, and G. Z. Yang, Adv. Opt. Mater. 5, 33 (2017).

8. T. Hayakawa, S. Fukada, and F. Arai, IEEE Trans. Robot. Autom. 30, 59 (2014).

9. D. Palima, A. R. Bañas, G. Vizsnyiczai, L. Kelemen, P. Ormos, and J. Glückstad, Opt. Express 20, 2004 (2012).

10. P. Galajda and P. Ormos, Appl. Phys. Lett. 78, 249 (2001).

11. S. L. Neale, M. P. Macdonald, K. Dholakia, and T. F. Krauss, Nat. Mater. 4, 530 (2005).

12. K. Ladavac and D. G. Grier, Opt. Express 12, 1144 (2004).

13. M. J. Villangca, D. Palima, A. R. Bañas, and J. Glückstad, Light Sci. Appl. 5, e16148 (2016).

14. D. Boyer, P. Tamarat, A. Maali, B. Lounis, and M. Orrit, Science 297 1160 (2002).

15. C. Loo, A. Lin, L. Hirsch, M. H. Lee, J. Barton, N. Halas, J. West, and R. Drezek, Technol. Cancer Res. Treat. 3, 33 (2004).

16. R. Rodríguez-Oliveros and J. A. Sánchez-Gil, Opt. Express 20, 621 (2012).

17. J. C. Ndukaife, A. V. Kildishev, A. G. A. Nnanna, V. M. Shalaev, S. T. Wereley, and A. Boltasseva, Nat. Nanotechnol. 11, 53 (2015).

18. M. Braun and F. Cichos, ACS Nano 7, 11200 (2013).

19. M. Essone Mezeme and C. Brosseau, Phys. Rev. E 87, 012722 (2013).

20. P. J. Rodrigo, V. R. Daria, and J. Glückstad, Opt. Express 12, 1417 (2004).

21. P. J. Rodrigo, L. Gammelgaard, P. Bøggild, I. Perch-Nielsen, and J. Glückstad, Opt. Express 13, 6899 (2005).

22. A. Ashkin, J. M. Dziedzic, J. E. Bjorkholm, and S. Chu, Opt. Lett. 11, 288 (1986).

23. N. Tarantino, J. Y. Tinevez, E. F. Crowell, B. Boisson, R. Henriques, M. Mhlanga, F. Agou, A. Israël, and E. Laplantine, J. Cell Biol. 204, 231 (2014).

24. G. Baffou, J. Polleux, H. Rigneault, and S. Monneret, J. Phys. Chem. C 118, 4890 (2014)

25. S. Duhr and D. Braun, Proc. Natl. Acad. Sci. USA 103, 19678 (2006).

26. Z. Kang, J. Chen, S.-Y. Wu, and H.-P. Ho, RSC Adv. 5, 105409 (2015).

27. H. Qian, M. P. Sheetz, and E. L. Elson, Biophys. J. 60, 910 (1991).

28. X. Michalet, Phys. Rev. E 82, 041914 (2010).

29. G. Dunderdale, S. Ebbens, P. Fairclough, and J. Howse, Langmuir 28 , 10997 (2012).

30. J. S. Donner, G. Baffou, D. McCloskey, and R. Quidant, ACS Nano 5, 5457 (2011).

31. B. J. Roxworthy, A. M. Bhuiya, S. P. Vanka, and K. C. Toussaint, Nat. Commun. 5, 3173 (2014).

\section{5}

306

307

308

309

310

311

312

313

314

315

316

317

318

319

320

321

322

323

324

325

326

327

328

329

330

331

332

333

334

335

336

337

338

339

340

341

342

343

344

345

346

347

348

349

350

351

352

353

354

355

356

357

358

359

360

361

362 


\section{Queries}

1. AU: Please review the edits made in the title.

2. AU: Your submitted paper listed two corresponding authors. However, OSA policy indicates that only one author may be designated the corresponding author. Please let us know the sole corresponding author and the e-mail address of the other author listed with an "e-mail:" label is correct. You also have the option of listing no corresponding author at all, in which case both of the e-mail addresses can be listed with the "e-mail:" label.

3. AU: As per journal requirement, section headings are not allowed in OL articles. Please check and confirm.

4. AU: The funding information for this article has been generated using the information you provided to OSA at the time of article submission. Please check it carefully. If any information needs to be corrected or added, please provide the full name of the funding organization/institution as provided in the CrossRef Open Funder Registry (https://search.crossref.org/funding).

\section{ORCID Identifiers}

The following ORCID identifiers were supplied for the authors of this article. Please review carefully. If changes are required, or if you are adding IDs for authors that do not have them in this proof, please submit them with your corrections for the article. Authors who do not have iDs on the proof may add them by logging into their OSA account. To do this, click on the "Update Account" link on your Prism homepage or log-in directly to http://account.osa.org, then click the button "Create or Connect your ORCID iD" in the ORCID section of the Participation tab. If the ORCID window does not appear, then change your browser settings to enable pop-ups. Please indicate in your corrections if you or any coauthors have done this. Each individual author is responsible for adding or correcting his or her $\mathrm{iD}$ using the steps outlined above.

- Einstom Engay https://orcid.org/0000-0002-2415-0316

- Ada-Ioana Bunea https://orcid.org/0000-0003-1273-2885 